\title{
Publicidad de bebidas alcohólicas. Algunas razones para establecer unos límites
}

\author{
SÁNCHEZ, R. \\ Federación de Asociaciones de Consumidores y Usuarios de Andalucía (FACUA) \\ Enviar correspondencia a: Rubén Sánchez García. Departamento de Control y Análisis de la Publicidad. Federación de Asociaciones de \\ Consumidores y Usuarios de Andalucía (FACUA). Bécquer, 25 A. 41002 Sevilla. Teléfono 954900 078. Fax: 954387852. \\ E-Mail: comunicacion@facua.org
}

\section{RESUMEN}

Este trabajo analiza las técnicas publicitarias utilizadas por la industria de las bebidas alcohólicas. En él se exponen ejemplos de los métodos empleados por los anunciantes para atraer a niños y adolescentes al consumo de alcohol y algunas conclusiones de estudios realizados sobre el sector y la percepción que tienen los consumidores de sus mensajes comerciales

Sexo, éxito y diversión son los mensajes que traslada a los jóvenes la industria de las bebidas alcohólicas en sus anuncios, que también presenta en ocasiones su producto como un medio para solucionar problemas. Una publicidad que en muchos casos atenta contra la dignidad de la mujer presentándola como un simple objeto sexual.

En el artículo también se pone de manifiesto la pasividad de las administraciones públicas en el debido control de las prácticas publicitarias ilícitas y la falta de una legislación específica que regule la publicidad de este producto. Normas que la industria se preocupa de frenar presentando una engañosa imagen de autorregulación y asegurando que sus anuncios no pretenden de ningún modo captar nuevos consumidores.

Junto a los mensajes engañosos y discriminatorios de la publicidad de bebidas alcohólicas, los anunciantes también recurren a prácticas prohibidas como la publicidad enmascarada en producciones cinematográficas y televisivas o la utilización de productos pretexto para promocionar en televisión bebidas de alta graduación.

Palabras clave: publicidad, alcohol, prevención, economía, autorregulación, administración, consumidores y/o asociaciones de consumidores.

\section{SUMMARY}

This work analyses the advertising and publicity techniques used by the alcoholic beverages industry. It provides examples of the methods employed by the advertisers to attract children and adolescents to the consumption of alcohol, some conclusions from studies made of the sector and consumer perception that consumers of these marketing messages.

Sex, success and having fun are the messages that are transmitted by the alcoholic beverages industry in its advertising aimed at the young and, on occasion, it also presents its product as a means of solving problems. In many cases, its advertising is an offence against the dignity of women by portraying them as merely sexual objects.

The article also draws attention to the passive attitude of public administrations adequately monitoring illicit advertising and publicity practices, and to the lack of specific legislation to regulate the advertising of this product; regulation that the industry is intent on preventing by presenting a deceptive image of self regulation, and insisting that their advertisements do not attempt to attract new consumers, in any way.

Together with the deceptive and discriminatory messages of alcoholic beverage advertising, the advertisers also resort to prohibited practices such as publicity concealed in film and television productions or to the utilisation of pretext products to promote beverages with a high alcoholic content on television.

Key words: alcohol, publicity, enterprises, prevention, self regulation, government control, consumers and/or consumers associations. 
$\mathbf{L}$ os jóvenes son el principal objetivo de la industria del alcohol. Su publicidad explota sus mayores deseos. Les ofrece sexo, éxito, diversión... Una vida ideal en la que ellos son el centro de todo. Los protagonistas de estos anuncios son atractivos, atléticos, divertidos, prototipos que despiertan el deseo de emulación. La bebida, evidentemente, forma parte de todo ese mundo perfecto, en el que se elimina cualquier preocupación respecto a la salud. Mientras, los límites a la publicidad de alcohol son escasos, y ni el Ministerio de Sanidad y Consumo ni las Administraciones competentes de las Comunidades Autónomas ponen especial empeño en hacer cumplir las pocas restricciones existentes.

La publicidad genera una actitud positiva hacia el alcohol que hace olvidar que, al fin y al cabo, es una droga. Una droga extremadamente peligrosa que usada sin moderación puede destruir por completo a sus consumidores, muy sensibles, especialmente en el caso de los adolescentes, a los engañosos mensajes que transmiten estos anuncios. Sin embargo, las restricciones legales a la publicidad de bebidas alcohólicas son pocas y ambiguas. La industria sostiene que sus anuncios son inocuos y el Gobierno no se preocupa demasiado por llevarle la contraria.

La publicidad de bebidas alcohólicas se asocia a "un amplio conjunto de valores positivos; en primer lugar la armonía, la sociabilidad y el liderazgo, seguidos de la alegría, el placer, la diversión, el éxito y el estilo", según un estudio realizado en 1994 por la empresa de investigación de mercados Contexto sobre anuncios de estos productos en televisión y que podría extrapolarse perfectamente a otros medios de comunicación.

Según este estudio, los anuncios televisivos de alcohol "reproducen los hábitos y modos de ingesta juveniles (los bares, la calle, la música, el grupo de amigos)". En ellos "se hace más hincapié en los valores simbólicos que en los valores de uso; es decir, se habla más del prototipo del bebedor que de la bebida en sí, haciendo hincapié en el significado del alcohol como indicador de pertenencia a un grupo de referencia".

La Asociación de Distribuidores e Industrias de Grandes Marcas de Bebidas (ADIGRAM) afirma' que la publicidad "no es la causa del problema" ya que "no contiene mensajes que inciten a la irresponsabilidad o abuso en el consumo". "En muchos casos (los adolescentes) encuentran en el alcohol un medio para el reconocimiento y la integración dentro del grupo de amigos, ya que suelen asociar el hecho de soportar mucho alcohol con una especial "fortaleza." A esto se añade que, a esta edad, se cuestiona la autoridad y la dependencia de los padres y contemplan la bebida como una forma de emancipación y rebeldía".

¿Qué significan entonces eslóganes como "Be Free. Be Beefeater" (Sé libre. Sé Beefeater); "Beefeater: Unidos por lo bueno"; "Larios: Es lo que vives"; o "Los amigos nunca son casuales?". Campari. Nunca es casual.

Según una encuesta ${ }^{2}$ realizada por estudiantes de la Politécnica de Hatfield (Gran Bretaña), los jóvenes describen el whisky, por ejemplo, como "la bebida principal de los hombres maduros convencionales, triunfadores, extrovertidos y dominantes"; el vino es una bebida con sabor "relajante" para "personas modernas, de éxito y cultivadas".

La tercera parte de los jóvenes españoles gasta el $90 \%$ de su dinero en bebidas alcohólicas, según la Fundación de Ayuda a los Toxicómanos de la Cruz Roja.

\section{SEXOY DISCRIMINACIÓN}

Una sensual joven de piel tostada mira fijamente al espectador, que desearía ver cómo la chica se lleva a los labios ese vaso lleno de ron con el que cubre un pecho desnudo. "Ron Negrita. Sabores caribeños". No hay que ser muy perspicaz para darse cuenta del otro sabor al se refiere el anuncio... además del ron.

En 1988, la Facultad de Psicología de la Universidad Autónoma de Madrid, realizó un 
análisis sobre publicidad en prensa que reveló cómo en el $23,9 \%$ de los casos la recompensa que se ofrece al consumidor de la bebida anunciada es la satisfacción sexual (seguida del prestigio social, en un $13 \%$, y de la amistad, en el 4,3\%). En el $40 \%$ de los anuncios la mujer aparece como objeto sexual. De hecho, es en la publicidad de bebidas alcohólicas donde la mujer aparece en mayor proporción ofrecida como un objeto de consumo más.

Un anuncio del whisky Ballantine's muestra el vientre de una mujer con un banderín con el número dieciocho tatuado debajo del ombligo y la cremallera de sus vaqueros semiabierta. Debajo de esta imagen, una botella de Ballantine's, con la forma de una bolsa, llena de palos de golf. El significado es claro, la botella contiene el instrumento (los palos, el whisky) para lograr el triunfo, llegar al último hoyo del campo, alcanzar el éxito sexual.

La frontera de la publicidad engañosa al utilizar reclamos sexuales en los mensajes comerciales se traspasa cuando en ellos se reduce a la mujer a un mero objeto pasivo destinado a satisfacer los deseos del hombre. Un mensaje censurable, pero también peligroso cuando se combina con el alcohol. ¿Cómo puede lanzar la idea de que las mujeres son más fáciles de conseguir cuando se está bajo los efectos del alcohol?

Un anuncio de los años 80 para la promoción de la ginebra Seagram mostraba a una mujer tumbada sobre un hombre, apretando la cabeza de él sobre su pecho. El texto que acompañaba a la imagen no podía ser más explícito: "Ellos dicen que es la ginebra número uno de América... Dicen también que puede transformar un 'tal vez' en un... 'otra vez'".

En un spot del licor de guindas Miura, un joven vampiro ofrece la bebida a una chica, para después morderle el cuello. Satisfecha, la chica vuelve a pedir "otro Miura" cuando se le acerca un nuevo joven.

Hay quien incluso sugiere que para que él tenga sexo, es ella quien debe beber. "Seguramente, ésta es la única ocasión en que desearás que acaben con tu botella de White
Label". El anuncio al que pertenece esta frase muestra un vaso con whisky y hielo en cuyo borde se ven las marcas de carmín que han dejado unos labios. Sobran comentarios.

Aunque la mujer es la víctima de la inmensa mayoría de los anuncios discriminatorios, y evidentemente no sólo de bebidas alcohólicas, no siempre ocurre así. En un anuncio de Martini emitido en 1997 que transcurre en la piscina de un hotel, el protagonista se percata de que la intención de una mujer que le ha invitado a su habitación no es otra que asesinarle, por lo que decide marcharse y le tira las llaves a un joven que toma el sol en el agua. Aunque ya de por sí el spot dejaba bastante clara su homosexualidad, Bacardí-Martini España publicó un anuncio en prensa en el que se aludía peyorativamente a la "dudosa masculinidad" del hombre de la piscina. El anuncio fue denunciado por FACUA, y aunque el anunciante negó en rotundo que contuviese el más mínimo atisbo de homofobia, ésta fue la única de las historias del hombre Martini, realizadas por la agencia McCannErickson, que no volvió a ser emitida el año siguiente.

Y la discriminación no sólo puede estar relacionada con el sexo. A finales de 1999, FACUA denunció a la empresa Domecq por dos anuncios que presentan ideales de pureza y perfección que, aun sin ser su intención, podían resultar ofensivos para los grupos étnicos que no se asemejen a ellos, al basarse en el color de la piel, el pelo y los ojos. Un anuncio de la ginebra Beefeater, de Allied Domecq, en el que se muestra a una mujer de ojos claros, piel y pelo blanco, utiliza el eslogan "Be Pure" (sé pura).La presentación del estereotipo de mujer pura basado en los citados rasgos remite a unos cánones de belleza que pueden degradar a otros que no coincidan con ellos y resultar racistas.

\section{NIÑOS}

Los niños son, directa o indirectamente, víctimas de la publicidad del alcohol. Un estudio realizado a finales de los 80 por la Univer- 
sidad de Edimburgo (Escocia) registró un notable aumento en la proporción de anuncios televisivos de bebidas alcohólicas entre las 18:00 y las 19:00 horas, cuando hay un alto porcentaje de niños entre los telespectadores, lo que concluyó que no sólo no se hacían esfuerzos por alejar la publicidad directa de los jóvenes, sino que "sugieren la posibilidad de que se esté tratando justamente de influir preferentemente en ellos".

En España, lo menores de dieciséis años (dieciocho en algunas comunidades autónomas) no pueden, al menos legalmente, consumir bebidas alcohólicas. La industria lo sabe, pero naturalmente no puede evitar que muchos menores sean seducidos por esos anuncios en los que el bebedor es el líder del grupo, el que más éxito tiene con el sexo opuesto...

Generalmente, la cerveza es la bebida de iniciación, el primer paso hasta llegar a la ginebra, el vodka, el ron o el whisky. Pero están apareciendo refrescos con una graduación alcohólica muy baja, como Locura Lemon, con los que se atrae a los jóvenes más reacios... y también más niños.

\section{LOS GRANDES BEBEDORES}

"El 32\% de los bebedores de cerveza beben el $80 \%$ de la cerveza... El $14 \%$ de las personas que beben ginebra consumen el $80 \%$ del total. Cualquiera que sea el sector en que te muevas, no pierdas de vista a los grandes consumidores del producto". La afirmación es del famoso publicista David Ogilvy. Tal vez sobren comentarios.

"El camino del éxito está sembrado de piedras. Déjenos allanarlo para usted", decía un anuncio de 1976 del whisky Johnnie Walker Etiqueta Negra. La Comisión Federal para el Comercio de EE. UU ${ }^{3}$ emitió un informe advirtiendo que el anuncio "ofrecía el producto como un medio para aliviar el estrés y la tensión derivados de la lucha por el éxito."Pero lo más importante, en dicho informe se señalaba que la investigación de estilos de vida encargada por la distribuidora de Johnnie Walker para la realización de la campaña publicitaria había aconsejado que se fijase como objetivo "a las personas que (en las encuestas de mercado) habían manifestado una identificación personal profunda con distintas actitudes asociadas a problemas con la bebida (como "No sé si podré llegar al final de la jornada sin tomar un trago")".

En España, un reciente anuncio del brandy 103, de Osborne, utiliza una frase casi calcado a la del anterior. "¿Espinas en tu vida? ¿Llévalas de forma suave?".

Aunque la industria lo niega, hay muchos anuncios que fomentan el consumo abusivo de alcohol. Frases como "¿Has hecho ya tu buena acción del día?" (White Label); "Después del curro, el mono" (Anís del mono); "¿No sería bonito que este mes todos los días fueran rojos?" (en un anuncio de Johnnie Walker Etiqueta Roja donde aparecía un calendario) o "Todo va bien con Licor 43" incitan a beber a diario o de forma irresponsable.

El abuso del alcohol es responsable de 20.000 muertes al año y causante de 13.000 accidentes de tráfico. Origina unos costes directos e indirectos de 637.717 millones de pesetas anuales. Las consecuencias de la adicción a esta droga absorben el equivalente al $16,2 \%$ del presupuesto sanitario público de este año. En el mundo laboral, origina más de 393.000 millones de pesetas en pérdidas. Los tratamientos de desintoxicación, a los que tan sólo se someten el $20 \%$ de los afectados, nos cuestan a los españoles 18.000 millones de pesetas de nuestros impuestos. El alcohol está detrás del $25 \%$ de los casos de violencia familiar, del $27 \%$ de los abusos a menores $y$, según otras fuentes, del $60 \%$ de los accidentes de tráfico que se producen en los fines de semana. En España hay en torno a 1.600.000 alcohólicos. El grueso de los adictos tiene entre 35 y 40 años, aunque el número de jóvenes enganchados es alarmante (según otras fuentes, uno de cada diez niños andaluces de 11 a 14 años se han emborrachado al menos dos veces en su vida).

La industria del alcohol no se siente responsable de estas cifras. "La publicidad no 
es la causa del problema", dicen, ya que sólo busca que los bebedores cambien de marca y no atraer a nuevos consumidores ni fomentar el consumo abusivo. Tampoco se sienten orgullosos de que su producto, mal utilizado, puede llegar a matar. Pero insisten en que ellos no tienen la culpa de la irresponsabilidad de algunos de sus clientes. Al fin y al cabo, su negocio no es la salud de los demás

\section{UNA AUTORREGULACIÓN POCO EFICAZ}

Ante el peligro de nuevas restricciones a su publicidad, la industria del alcohol ha tomado la iniciativa en muchos países desarrollando sus propios códigos de autorregulación. Con ello, la legislación publicitaria no sólo no avanza, sino que muchos gobiernos, con una irresponsabilidad que entre otras cosas pretende evitar conflictos con la industria, llegan a dejar en manos de los propios anunciantes el control publicitario.

En España, las denuncias presentadas por FACUA a finales de los 90 ante la Asociación para la Autorregulación de la Comunicación Comercial (AACC), una organización integrada por anunciantes, agencias de publicidad y medios de comunicación, no dijeron nada en favor del Código de la Asociación Española de Anunciantes para la Autorregulación de la Publicidad de Bebidas Alcohólicas. Así, por ejemplo, la Autocontrol desestimó las denuncias de FACUA contra la publicidad de White Label por fomentar el consumo abusivo, advirtiendo que las frases de sus anuncios ("El $70 \%$ del cuerpo es agua. Ya está bien ¿no?"y "Has hecho ya tu buena acción del día") eran humorísticas y que, al fin y al cabo, en los mismos se incluía el mensaje "Bebe con moderación. Es tu responsabilidad". También desestimó varias denuncias contra marcas de bebidas alcohólicas que utilizaban reclamos sexistas que atentaban contra la dignidad de la mujer, presentándola como un mero objeto sexual.

Autocontrol también desestimó una reclamación contra un anuncio gráfico de Ron Barceló en el que se muestra una mujer de espaldas, vestida con un tanga, en un paisaje de una paradisíaca isla caribeña, con el texto: "Ese oscuro objeto de deseo. Ron Barceló. Auténtico sabor dominicano". La resolución de Autocontrol se basaba entre otras cosas en que "la simple representación del cuerpo humano, ya sea desnudo o con sugerentes vestimentas, no puede ser entendida 'per se' como un atentado contra la dignidad de las personas", entendía que la mención "oscuro objeto de deseo" se refería a la bebida y no a la joven y no incurría en ningún tipo de discriminación y obviaba la expresión "auténtico sabor dominicano". El anuncio de Ron Barceló no sólo presenta a la mujer como un mero objeto sexual, sino que, indirectamente, también potencia el tan denostado turismo sexual en los países del caribe.

Autocontrol tampoco se pronunció en contra de otro anuncio, emitido en televisión, en el que un joven se convierte en el centro de atención de su grupo de amigos al pedir "un Baileys" en un bar de copas. En el spot, una chica se presta a besar al protagonista para conseguir probar el licor de sus labios. Para Autocontrol, el anuncio no mostraba el alcohol como un medio para lograr el éxito sexual, al entender que un beso en los labios no llega a ser una conquista sexual.

Curiosamente, la misma Autocontrol instó a la editorial $\mathrm{G}+\mathrm{J}$ a retirar de las cadenas un anuncio de su revista Cosmopólitan en el que una joven que hace ejercicio en una bicicleta estática simula un orgasmo jadeando y moviéndose bruscamente al incrementar el ritmo de sus pedaladas. "Descubre uno de los deportes más sanos y estimulantes: el autoerotismo", rezaba el anuncio de la revista, en cuya portada podía leerse: "Aprende tocando: Masturbarse ya no es tabú". Para Autocontrol, el anuncio no respetaba "los criterios imperantes del buen gusto, el decoro social y las buenas costumbres" y podía faltar "al debido respeto a las convicciones religiosas", además de perjudicar seriamente "el desarrollo moral de niños y adolescentes en la opinión de un considerable sector de la opinión pública". 
"Bebe con moderación. Es tu responsabilidad". Esta advertencia, que los miembros de la Asociación de Distribuidores e Industrias de Grandes Marcas de Bebidas (ADIGRAM) incluyen, por iniciativa propia, en sus anuncios, es prácticamente inapreciable por el consumidor, dado su minúsculo tamaño y el hecho de que en algunos casos esté impreso en colores que hacen aún más difícil que se repare en su existencia.

Así lo pone de manifiesto un estudio sobre publicidad de bebidas alcohólicas en prensa realizado por FACUA, que reveló que la citada leyenda era hasta 510 veces más pequeña que la superficie total de los anuncios. Pretende realmente ADIGRAM hacer una llamada a la moderación o simplemente intenta evitar nuevas restricciones a la publicidad de bebidas alcohólicas?

FACUA considera que el Ministerio de Sanidad y Consumo debe establecer la obligatoriedad de incluir un mensaje en los anuncios de bebidas alcohólicas en el que se advierta de los peligros del consumo abusivo, el cuál debería ser perfectamente visible por el consumidor.

\section{“NO BUSCAMOS NUEVOS CONSUMIDO- RES"}

"Por increíble que pueda parecer, dados los miles de millones de dólares que alimentan la publicidad del alcohol, hay una importante corriente de opinión (que en parte se nutre también de capital empresarial) que sostiene que esas colosales sumas gastadas en publicidad no ejercen ningún impacto en persuadir a nuevos consumidores a engrosar el mercado del alcohol. Una miríada de monografías que recogen investigaciones ostensiblemente "científicas," muchas de ellas basadas en técnicas económicas, estudian la relación de la publicidad con el consumo en varios países. Debe investigarse cuidadosamente cuál es la fuente de financiación de esos estudios, porque en varios es la red de influencias del alcohol la veces por intermedio de sus asociacio- nes empresariales) la que directa o indirectamente financia esas monografías. No debería sorprender a nadie que lo que fue aclamado como un notable estudio, cuyas austeras conclusiones eran que "no existe ninguna evidencia de que la publicidad de bebidas alcohólicas tenga un impacto significativo en el abuso del alcohol," estuviera patrocinado por la Asociación de Cerveceros de Estados Unidos".

Este texto ${ }^{4}$, recogido por el periodista norteamericano Eric Clark en su libro La publicidad y su poder, pertenece a un extenso dossier sobre el marketing del alcohol realizado por la Organización Mundial de la Salud y que nunca fue publicado, según se dice, por las presiones de la industria.

\section{PUBLICIDAD ENCUBIERTA}

Según un estudio realizado en 1994 por la consultora Contexto, "el medio que vehicula más claramente la relación publicitaria entre el alcohol y los menores es la radiofórmula, por su especial penetración entre éstos". "La implicación de los locutores y disk-jokeys", continúa el estudio, "en la publicidad de las bebidas alcohólicas es absoluta en muchas emisoras, jugando así un papel de prescriptores activos: mezclan la presentación de los discos con los mensajes promocionales, crean un ambiente de complicidad en torno al alcohol con los oyentes, asocian de modo inextricable la marcha, la diversión y el alcohol".

El brandy ya no es sólo "cosa de hombres". El Consejo Regulador del Brandy de Jerez puso en marcha hace unos años una campaña para fomentar su consumo entre los jóvenes. Para conseguirlo, recurrió a un método muy eficaz, pero también ilegal: la publicidad encubierta. Pásatelo a lo brandy o Esto es brandyoso son dos de las expresiones que utilizaron en sus programas locutores como José Antonio Abellán, José María García y Gomaespuma para introducir la palabra brandy en el lenguaje coloquial.

El estudio de Contexto también señala que "en muchos casos, las referencias al consu- 
mo de alcohol en las letras de las canciones emitidas contribuyen a reforzar ese peligroso lazo". La campaña del brandy incluirá una canción, que el grupo Ketama presentó en uno de sus discos, dedicada a esta bebida. Y no sólo influyen las letras, sino también las acciones, como que el cantante Loquillo se beba una botella de whisky en sus conciertos.

Los actores también influyen notablemente en la conducta de los jóvenes. Sylvester Stallone pide "un J\&B" en El Especialista y Michael Douglas se toma un Jack Daniel's después de hacer el amor con Sharon Stone en Instinto Básico. ¿Casual? Posiblemente tanto como que, generalmente, en películas que ofrecen una imagen negativa del alcohol no aparezcan las marcas de las bebidas. Casi dos de cada diez películas incluyen la presencia de alguna marca de bebidas alcohólicas, según reveló un estudio realizado por FACUA $^{5}$. En cuanto a la publicidad encubierta en las teleseries españolas ${ }^{6}$, el 8,3\% de los productos son marcas de alcohol, la mayoría con más de veinte grados.

\section{ANUNCIANDO LO PROHIBIDO}

La publicidad de productos como el tabaco, las bebidas alcohólicas o ciertos medicamentos ha sido vetada en algunos medios de comunicación por muchos gobiernos. Sin embargo, numerosas compañías se resisten a acatar las restricciones y recurren a todo tipo de trucos para anunciarse ante la mirada pasiva de las autoridades competentes.

La publicidad indirecta consiste en presentar el nombre, la forma, el eslogan o incluso tan sólo el color del producto pero sin mencionar directamente que se trata del producto de publicidad prohibida. Son productos pretexto como los combinados Cuba Libre Bacardí y Soberano Mixer. En España, la publicidad indirecta se utiliza desde que la Ley General de Publicidad de 1988 prohibió anunciar por televisión tabaco y bebidas alcohólicas con más de veinte grados.

A inicios de 1996, las cadenas emitieron un anuncio de Bacardí en el que no se mencio- naba ningún producto. Sin embargo, en él podía oírse un sonido similar al de unos cubitos de hielo cuando caen en un vaso. En otro spot, de Ponche Caballero, la palabra ponche se sustituyó por otra de sonido similar, ponte; la botella aparecía en pantalla durante unos breves instantes.

En 1997, varias cadenas emitieron un anuncio en el que unas voluptuosas mujeres dejaban ver parte de sus encantos mientras que aparecía en pantalla el eslogan "Soberano. ¡Alegría!." Para justificar la emisión del spot, un pequeño rótulo indicaba que el Soberano anunciado no era una bebida de alta graduación, sino un -inexistente- licor de 12 grados. La misma argucia fue utilizada en esas fechas en un spot de Veterano. Aunque se seguía utilizando el logotipo y eslogan - "el toro"- que han identificado siempre al brandy de 36 grados de las bodegas Osborne, la empresa argumentaba que el Veterano anunciado era "un licor de brandy de 17,5 grados."

Hay compañías que incluyen en sus presupuestos publicitarios las multas que les podrán imponer por incumplir las prohibiciones. Pero, en la mayoría de los casos, los anunciantes de productos de publicidad prohibida aprovechan la pasividad de las autoridades para saltarse a su antojo las restricciones.

El product placement es una técnica de publicidad encubierta que consiste en emplazar estratégicamente un producto en el guión de una obra, por ejemplo una película o una serie de televisión, gracias a la cual los fabricantes de productos de publicidad prohibida pueden hacer caso omiso de las leyes sin que las autoridades, muy poco dadas a controlar estas prácticas, hagan nada por impedírselo.

Desde 1988, la publicidad encubierta en España no está permitida, pero tampoco controlada. Cinemarc, una agencia especializada en product placement, utiliza la misma ley que prohíbe la publicidad encubierta en la televisión (la Ley de Televisión sin Fronteras) para argumentar a sus clientes potenciales las posibilidades de que una película se emita en alguna cadena "ya que el $20 \%$ de las películas que emiten las televisiones debe corresponder a producciones españolas". 
En 1936, los miembros del Consejo de Alcoholes Destilados de EE.UU. acordaron voluntariamente no anunciarse a través de la radio y doce años después extendieron este compromiso a la televisión. Sin embargo, muchas marcas llevan años incumpliendo esta autorregulación mediante técnicas de publicidad encubierta. En un episodio de la serie de televisión Expediente $X$, el director adjunto del FBI Walter Skinner intenta ahogar sus penas con una botella de J\&P (i?), cuya forma y etiqueta es idéntica a la del whisky que todos conocemos.

En España, más del 7\% de los productos que aparecen en las teleseries de producción nacional son marcas de cigarrillos o de bebidas alcohólicas de alta graduación, según un estudio realizado por FACUA entre 1994 y 1999. Quince marcas de tabaco y dieciséis de bebidas con más de veinte grados han aparecido en treinta y seis teleseries emitidas en nuestro país. El Súper (Telecinco) es la serie donde se detectaron más marcas de alcohol de alta graduación.

Desde el inicio de las limitaciones a su publicidad, las compañías licoreras y tabaqueras patrocinan todo tipo de acontecimientos deportivos para que los consumidores asocien sus productos a la salud y a la buena forma física. De paso, la retransmisión de estas competiciones les permite anunciarse gratis en televisión esquivando las restricciones.

También con las vallas publicitarias de los estadios se pueden cometer irregularidades. "Me contaba un amigo, que fue jefe de publicidad de unas bodegas de Jerez, que pagaba con fino a ciertos cámaras para que enfocaran más tiempo la marca de la empresa", explica el publicista Amado Juan de Andrés en su libro Mecenazgo \& Patrocinio ${ }^{7}$.
Hay fabricantes de bebidas alcohólicas y de tabaco que han creado sus propios acontecimientos deportivos o culturales, bautizados con los nombres de sus productos, que en ocasiones se utilizan para anunciarlos indirectamente en televisión. En los últimos años, los espectadores españoles han podido ver en televisión las retransmisiones -y los anuncios- del Campeonato de España J\&B de Voley Playa, la Vuelta Beefeater de motos acuáticas y los Premios Smirnoff de Moda para Jóvenes Diseñadores, que curiosamente se crearon un año después de la promulgación de la Ley General de Publicidad.

\section{REFERENCIAS BIBLIOGRÁFICAS}

(1) ADIGRAM (Asociación de Distribuidores de Grandes Marcas de Bebidas), informe sobre su posición y actuaciones sobre el consumo de bebidas alcohólicas, Madrid, 1997.

(2) CLARK, ERIC. La publicidad y su poder, primera edición, Barcelona, Planeta, 1989, página 360.

(3) CLARK, ERIC. La publicidad y su poder, primera edición, Barcelona, Planeta, 1989, página 373.

(4) CLARK, ERIC. La publicidad y su poder, primera edición, Barcelona, Planeta, 1989, páginas 389-390.

(5) SÁNCHEZ GARCÍA, RUBÉN y CUTIÑO RIAÑO, JOSÉ CARLOS, Control y Análisis de la Publicidad 1995, Sevilla, FACUA, 1995.

(6) SÁNCHEZ GARCÍA, RUBÉN y CUTIÑO RIAÑO, JOSÉ CARLOS, Control y Análisis de la Publicidad 1999, Sevilla, FACUA, 1999.

(7) DE ANDRÉS, AMADO JUAN, Mecenazgo \& Patrocinio: Las claves del marketing del siglo XXI, Madrid, Editmex, 1993. 